\title{
Aging underlies heterogeneity between comorbidity and multimorbidity frameworks
}

\author{
Marco Vincenzo Lenti ${ }^{1}$. Catherine Klersy ${ }^{2}$ - Alice Silvia Brera ${ }^{1}$ Alessia Ballesio ${ }^{1}$. Gabriele Croce ${ }^{1}$. Lucia Padovini ${ }^{1}$. \\ Rachele Ciccocioppo ${ }^{1}$. Giampiera Bertolino ${ }^{1}$. Antonio Di Sabatino ${ }^{1}$. Gino Roberto Corazza ${ }^{1}$ (1)
}

Received: 29 July 2021 / Accepted: 17 November 2021 / Published online: 7 January 2022

(c) The Author(s), under exclusive licence to Società Italiana di Medicina Interna (SIMI) 2021

\begin{abstract}
Studies exploring differences between comorbidity (i.e., the co-existence of additional diseases with reference to an index condition) and multimorbidity (i.e., the presence of multiple diseases in which no one holds priority) are lacking. In this single-center, observational study conducted in an academic, internal medicine ward, we aimed to evaluate the prevalence of patients with two or more multiple chronic conditions (MCC), comorbidity, or multimorbidity, correlating them with other patients' characteristics. The three categories were compared to the Cumulative Illness Rating Scale (CIRS) comorbidity index, age, gender, polytherapy, 30-day readmission, in-hospital and 30-day mortalities. Overall, 1394 consecutive patients (median age 80 years, IQR 69-86; F:M ratio 1.16:1) were included. Of these, 1341 (96.2\%; median age 78 years, IQR 65-84; F:M ratio 1.17:1) had MCC. Fifty-three patients (3.8\%) had no MCC, $286(20.5 \%)$ had comorbidity, and 1055 (75.7\%) had multimorbidity, showing a statistically significant $(p<0.001)$ increasing age trend (median age 38 years vs 71 vs 82 , respectively) and increasing mean CIRS comorbidity index ( $1.53 \pm 0.95$ vs $2.97 \pm 1.43$ vs $4.09 \pm 1.70$, respectively). The CIRS comorbidity index was always higher in multimorbid patients, but only in the subgroups 75-84 years and $\geq 85$ years was a significant $(p<0.001)$ difference ( 1.24 and 1.36, respectively) noticed. At multivariable analysis, age was always independently associated with in-hospital mortality $(p=0.002), 30$-day mortality $(p<0.001)$, and 30-day readmission $(p=0.037)$, while comorbidity and multimorbidity were not. We conclude that age determines the most important differences between comorbid and multimorbid patients, as well as major outcomes, in a hospital setting.
\end{abstract}

Keywords Chronic disease $\cdot$ Clinical complexity $\cdot$ Multiple chronic conditions $\cdot$ Polypharmacy

\section{Introduction}

Although the prevalence of patients with multiple chronic conditions (MCC) is rising worldwide $[1,2]$, research on this topic still needs to fill in some important gaps of knowledge [3, 4]. New standardized definitions are needed so as to allow comparisons and meta-analyses [5], and it has been proposed that the concepts of comorbidity and multimorbidity should not be used interchangeably and that a distinction is desirable $[2,3,6]$. This is not a matter of

Gino Roberto Corazza

gr.corazza@smatteo.pv.it

1 Department of Internal Medicine, Clinica Medica, Fondazione IRCCS Policlinico San Matteo, University of Pavia, Viale Golgi 19, 27100 Pavia, Italy

2 Clinical Epidemiology and Biometry, Fondazione IRCCS Policlinico San Matteo, University of Pavia, Pavia, Italy semantics, given that the lack of a clear distinction has hampered the development of high-quality studies addressing these issues [2, 7]. In line with this view, specific Medical Subject Headings (MeSH) were recently designated to distinguish comorbidity from multimorbidity, which are now being used in scientific search engines as different MeSH $[8,9]$. More in detail, comorbidity indicates the combined effects of additional conditions in reference to a single index chronic disease under study, whereas multimorbidity is defined as the co-occurrence in the same individual of multiple diseases in which no single disease holds priority $[10,11]$. So, while comorbidity focuses on a single condition among many, multimorbidity describes the disease burden as a whole, and it has been theorized that this distinction may translate into specific treatment choices [12]. For instance, different principles of exercise have recently been outlined for patients with either comorbidity or multimorbidity [13]. There is no doubt, however, that in real life some overlaps 
may be unavoidable, and that a sharp distinction will have to be based more on ad hoc studies rather than on systematic reviews. In this regard, we herein aim at classifying MCC into comorbidity or multimorbidity frameworks and correlating the results with demographic and clinical outcomes in a large series of patients consecutively admitted to an academic internal medicine ward. The significance of differentiating comorbidity from multimorbidity will then be discussed.

\section{Material and methods}

Data from consecutively enrolled adult patients in the San MAtteo Complexity (SMAC) study, a large ongoing prospective research project on clinical complexity (NCT03439410) conducted in November 2017-November 2019 at our Institution, were analyzed. The follow-up of these patients is still ongoing, with the aim of validating a recently developed clinical complexity index [14-16].

The San Matteo Hospital Foundation, an academic hospital of Northern Italy, serves a population of $\sim 550,000$ people, and most patients (>90\%) access the Emergency Department before admission. In the SMAC study, adult patients admitted to our internal medicine unit were included, regardless of the cause of admission. The details of the enrolment have already been described elsewhere [17]. Demographic (i.e., age, gender) and clinical data (i.e., polypharmacy, body mass index [BMI], frailty according to the Edmonton Frail Scale [18], length of stay, in-hospital and 30-day mortalities, 30-day readmission) were collected, including the Cumulative Illness Rating Scale (CIRS) [19], a common tool for assessing MCC in clinical studies [20]. Assuming that the correct selection of such a tool should depend on the outcomes under investigation [20,21], we embraced the CIRS because of its ability to predict mortality and hospital stay and readmission [22]. Diseases were identified and categorized according to the International Classification of Diseases (ICD; 9th revision). In particular, the whole ICD9 chapter was used for categorization (e.g., chapter 2, neoplastic diseases; chapter 3, endocrine, nutritional and immunological diseases; etc....).

All patients with MCC, defined as the simultaneous presence of at least two chronic conditions, were either classified into a comorbidity or a multimorbidity framework, strictly according to the MeSH definitions. Comorbidity was defined as the co-existence of additional diseases with reference to an index condition [8] while multimorbidity as the presence of multiple diseases in which no one holds priority [9]. Recognized complications of an index disease (e.g., ascites, hepatic encephalopathy in liver cirrhosis) were considered associated conditions in respect to an index disease, and thus classified as comorbidities [23].
The absence of chronic diseases, or the presence of only one chronic condition -also defined by the appropriate $\mathrm{MeSH}$ [24]- were categorized as "no MCC". Practically, an expert academic physician (MVL), who was unaware of patients' age, gender, and outcomes, partitioned all patients into one of these three categories, following the abovementioned criteria. All anonymous demographic and clinical data were retrieved from the dedicated REDCap database. As practical and representative examples, patients in whom (i) a univocal etiopathogenic link for disease clustering was noticed (e.g., alcohol abuse causing liver cirrhosis and its consequences, or obesity causing metabolic syndrome) or (ii) a clear clinical priority was evident (e.g., recent onset of a neoplastic disease in a patient with mild essential hypertension), were categorized as comorbid. Instead, patients with (i) unrelated MCC (e.g., asthma, autoimmune thyroid disease, Parkinson's disease) with no clear risk factors, or (ii) MCC with multiple risk factors (e.g., obesity, tobacco smoking, alcohol abuse), where categorized as having multimorbidity. Finally, cases in which it was not possible to clearly categorize the patient into one of the two categories (i.e., the actual presence of an index disease was uncertain), according to the $\mathrm{MeSH}$ criteria, were considered as having multimorbidity. Prior to study commencement, a random sample of 25 patients within each category were categorized and reviewed by three other physicians with different experience, to verify whether any discordance existed. The agreement among the observers was $>90 \%$.

As the primary aim, the three categories (no MCC, comorbidity, and multimorbidity) were compared to the CIRS comorbidity index. As a secondary aim, other correlations included age, gender, polytherapy, length of stay, CIRS severity index, 30-day readmission, in-hospital and 30 -day mortalities. The study was approved by the local ethics committee (Protocol number 2017/0019414) and all patients provided written informed consent. All data generated or analyzed during this study are included in this published article.

\section{Statistical analysis}

For the purpose of this study, the sample size was not calculated a priori. However, the sample size $(n=1000)$ was based on the primary aim of the SMAC study. Continuous data were described with mean and standard deviation or median and interquartile range (IQR; i.e., 25th-75th percentiles), and categorical data were described as counts and percent. They were compared between patients' categories with the Kruskall-Wallis test and the Fisher exact test, respectively. Data were analyzed with generalized linear models to adjust for age the association of patients' category with the CIRS comorbidity and severity indices. Differences between categories and $95 \%$ CI were retrieved from the model. The 
interaction of age and patients' categories was assessed and subgroup analyses by age categories were performed. Hospital mortality, 30-day mortality, and 30-day readmission were analyzed with logistic models. Multivariable models for these outcomes were fitted, where the choice of covariates was decided a priori based on clinical considerations. Among the covariates, we also included the index disease (comorbidity framework) or the reason for hospital admission (multimorbidity framework or no MCC) according to the main chapters of the International Classification of Diseases (ICD; 9th revision). A two-sided $p<0.05$ was considered statistically significant. For post-hoc comparisons, $p<0.017$ was set for significance (Bonferroni correction). All analyses were performed using Stata 16 (StataCorp, College Station, TX, USA).

\section{Results}

Overall, 1394 consecutive patients (median age 80 years, IQR 69-86; F:M ratio 1.16:1) enrolled in the SMAC study were included. Of these, 1341 (96.2\%; median age 78 years, IQR 65-84; F:M ratio 1.17:1) had two or more MCC and were categorized as either comorbid or multimorbid. Although the comparison between patients with MCC $(n=1341)$ and no MCC ( $n=53)$ was very unbalanced, significant differences were noticed (Supplementary Table 1). In brief, patients with MCC displayed a higher median age ( 80 vs 38 years), mean CIRS comorbidity index (3.85 vs $1.53)$, mean CIRS severity index (1.78 vs 1.32 ), polypharmacy ( $72.7 \%$ vs $0 \%)$, median length of stay (14 vs 9 days), and 30 -day mortality (12.9\% vs $3.8 \%$ ).

Demographic and baseline clinical characteristics of the entire cohort according to the presence of no MCC, comorbidity, or multimorbidity are reported in Table 1. Notably, most patients were categorized as having multimorbidity $(1055,75.7 \%)$, and a statistically significant $(p<0.001)$ increasing age was noticed, namely no MCC (median age 38 years), comorbidity (median age 71 years), and multimorbidity (median age 82 years). Moreover, the distribution of age groups significantly differed $(p<0.001)$ both among the three categories and within each category. In particular, the prevalence of multimorbidity significantly increases $(p<0.001)$ with increasing age, while the opposite significant trend $(p<0.001)$ was noticed for comorbidity. Although the median number of chronic conditions did not statistically differ between multimorbid (6, IQR 5-8) and comorbid patients (4, IQR 3-5), the CIRS comorbidity index (mean 4.09 vs 2.97 ) and the CIRS severity index (mean 1.82 vs $1.63)$ were significantly higher $(p<0.001)$ in multimorbid vs

Table 1 Demographic and baseline clinical characteristics of the 1394 patients included in the study according to the presence of no multiple chronic conditions (MCC), comorbidity, or multimorbidity

\begin{tabular}{|c|c|c|c|c|c|c|c|}
\hline & \multirow[b]{2}{*}{ No MCC (I) } & \multirow[b]{2}{*}{ Comorbidity (II) } & \multirow[b]{2}{*}{ Multimorbidity (III) } & \multirow[b]{2}{*}{$p$ value } & \multicolumn{3}{|c|}{ Post-hoc comparisons } \\
\hline & & & & & $p$ value (I vs II) & $\begin{array}{l}p \text { value } \\
\text { (I vs III) }\end{array}$ & $\begin{array}{l}\text { p-value } \\
\text { (II vs III) }\end{array}$ \\
\hline No. of patients, $n(\%)$ & $53(3.8)$ & $286(20.5)$ & $1055(75.7)$ & - & - & - & - \\
\hline Female gender, $n(\%)$ & $25(47.2)$ & $153(53.5)$ & $571(54.1)$ & 0.610 & - & - & - \\
\hline Age, median (years; IQR) & $38(30-57)$ & $71(57-81)$ & $82(75-87)$ & $<0.001$ & $<0.001$ & $<0.001$ & $<0.001$ \\
\hline Age groups, $n$ (years; \%) & & & & $<0.001$ & $<0.001$ & $<0.001$ & $<0.001$ \\
\hline $18-64$ & $42(79.3)$ & $105(36.7)$ & $116(11.0)$ & - & - & - & - \\
\hline $65-74$ & $4(7.5)$ & $59(20.6)$ & $142(13.5)$ & - & - & - & - \\
\hline $75-84$ & $6(11.3)$ & $81(28.3)$ & $398(37.7)$ & - & - & - & - \\
\hline$\geq 85$ & $1(1.9)$ & $41(14.4)$ & $399(37.8)$ & - & - & - & - \\
\hline CIRS comorbidity index, mean (SD) & $1.53(0.95)$ & $2.97(1.43)$ & $4.09(1.70)$ & $<0.001$ & $<0.001$ & $<0.001$ & $<0.001$ \\
\hline CIRS severity index, mean (SD) & $1.32(0.22)$ & $1.63(0.26)$ & $1.82(0.28)$ & $<0.001$ & $<0.001$ & $<0.001$ & $<0.001$ \\
\hline $\mathrm{EFS}>5, n(\%)$ & $51(3.6)$ & $115(8.2)$ & $268(19.2)$ & $<0.001$ & $<0.001$ & $<0.001$ & $<0.001$ \\
\hline Intake $\geq 5$ medications, $n(\%)$ & $0(0)$ & $145(50.9)$ & $830(78.7)$ & $<0.001$ & $<0.001$ & $<0.001$ & $<0.001$ \\
\hline BMI class, $n(\%)$ & & & & 0.003 & 0.069 & 0.004 & 0.043 \\
\hline$<18.5$ & $6(11.3)$ & $31(10.8)$ & $65(6.1)$ & - & - & - & - \\
\hline $18.5-24.9$ & $36(67.9)$ & $142(49.7)$ & $517(49.0)$ & - & - & - & - \\
\hline $25-29.9$ & $7(13.2)$ & $71(24.8)$ & 309 (29.3) & - & - & - & - \\
\hline$\geq 30$ & $4(7.6)$ & $42(14.7)$ & $164(15.6)$ & - & - & - & - \\
\hline
\end{tabular}

Significance after the Bonferroni correction is set at $p<0.017$. Post-hoc comparisons are in italics

$B M I$, Body Mass Index; $C I R S$, Cumulative Illness Rating Scale; EFS, Edmonton Frail Scale; $I Q R$, interquartile range; $M C C$, multiple chronic conditions; $S D$, standard deviation 
comorbid patients. Similarly, the intake of $\geq 5$ medications was significantly more prevalent $(p<0.001)$ in multimorbid vs comorbid patients (78.7\% vs 50.9\%). Differently, after the Bonferroni correction, the distribution of the BMI did not differ $(p=0.043)$ between comorbid and multimorbid patients.

The correlates of the CIRS comorbidity index with patients' categories are reported in Table 2. In all models, the category "no MCC" was considered as "base". In the unadjusted model, patients with multimorbidity had a significantly greater CIRS comorbidity index compared to those with comorbidity. We then assessed the interaction of age and patients' categories in a model including both the main effects and the interaction proving a significant effect modification $(p<0.001)$. For this reason, the association of the CIRS comorbidity index with patients' categories was assessed by age groups. When adjusting the analysis for age groups, despite the CIRS comorbidity index turning out to be always higher in multimorbid patients, only in the subgroups $75-84$ years and $\geq 85$ years was a significant difference ( 1.24 and 1.36, respectively; $p<0.001)$ noticed. The same trend was observed when using the CIRS severity index as the dependent variable (Supplementary Table 2). In particular, patients with multimorbid patients had a significantly greater CIRS severity index compared to comorbid patients and only in the subgroups 75-84 years and $\geq 85$ years was a significant difference $(0.51$ and 0.38 , respectively; $p<0.001)$ noticed.

The median length of stay was significantly lower (9 days, IQR 7-13) for patients with no MCC compared to the other groups $(p<0.001)$, while no difference was noticed between comorbid (14 days, IQR 9-23) and multimorbid (14 days, IQR 10-21) patients. Overall, 267 patients died, of whom 92 (6.6\%) during the hospital stay and $175(12.5 \%)$ within 30 days after discharge. Table 3 shows the odds ratio for in-hospital and 30-day mortality and 30-day readmission in patients with comorbidity compared to multimorbidity (no MCC was again considered as base). Of patients who died in hospital, 2 (3.8\%) had no MCC, 22 (7.7\%) had comorbidity, 68 (6.4\%) had multimorbidity $(p=0.527)$, while of patients who died within 30 days after discharge, 2 (3.8\%) had no MCC, 43 (15.0\%) had comorbidity, and $130(12.3 \%)$ had multimorbidity $(p=0.068)$. Additionally, 207 patients $(14.8 \%)$ were readmitted to the hospital within 30 days after discharge. Of these, 6 (11.7\%) had no MCC, 44 (22.7\%) had comorbidity, and $157(21.9 \%)$ had multimorbidity $(p=0.213)$. Hence, no differences emerged regarding in-hospital

Table 2 Correlates of the CIRS comorbidity index in the entire cohort of patients

\begin{tabular}{|c|c|c|c|c|c|c|}
\hline & $\begin{array}{l}\text { CIRS comorbidity } \\
\text { index (mean; SD) }\end{array}$ & $\begin{array}{l}\text { Difference } \\
\text { (vs no MCC) }\end{array}$ & $95 \% \mathrm{CI}$ & $p$ value & Multi- vs comorbidity & $p$ value \\
\hline Unadjusted model & & & & $<0.001$ & & \\
\hline No MCC & $1.53(0.95)$ & 0 & & & & \\
\hline Comorbidity & $2.97(1.43)$ & 1.44 & $1.13-1.74$ & $<0.001$ & & \\
\hline Multimorbidity & $4.09(1.70)$ & 2.56 & $2.29-2.84$ & $<0.001$ & $1.12(0.89-1.36)$ & $<0.001$ \\
\hline \multicolumn{7}{|c|}{ Subgroup analysis ( $p$ for interaction $<0.001$ ) } \\
\hline \multicolumn{7}{|c|}{ Subgroup for age $18-64(N=263)$} \\
\hline No MCC & & 0 & & & & \\
\hline Comorbidity & & 1.22 & $0.83-1.62$ & $<0.001$ & & \\
\hline Multimorbidity & & 1.66 & $1.23-2.08$ & $<0.001$ & $0.43(-0.007-0.12)$ & 0.93 \\
\hline \multicolumn{7}{|c|}{ Subgroup for age $65-74(N=205)$} \\
\hline No MCC & & 0 & & & & \\
\hline Comorbidity & & 1.81 & $1.17-2.44$ & $<0.001$ & & \\
\hline Multimorbidity & & 2.32 & $1.75-2.89$ & $<0.001$ & $0.52(-0.08-1.12)$ & 0.113 \\
\hline \multicolumn{7}{|c|}{ Subgroup for age $75-84(\mathrm{~N}=485)$} \\
\hline No MCC & & 0 & & & & \\
\hline Comorbidity & & 1.42 & $0.49-2.35$ & 0.003 & & \\
\hline Multimorbidity & & 2.66 & $1.75-3.57$ & $<0.001$ & $1.24(0.84-1.64)$ & $<0.001$ \\
\hline \multicolumn{7}{|c|}{ Subgroup for age $\geq 85(\mathrm{~N}=441)$} \\
\hline No MCC & & 0 & & & & \\
\hline Comorbidity & & 0.85 & $0.39-1.31$ & $<0.001$ & & \\
\hline Multimorbidity & & 2.22 & $2.06-2.38$ & $<0.001$ & $1.36(0.77-1.96)$ & $<0.001$ \\
\hline
\end{tabular}

Differences between categories are computed using a generalized linear model

$C I$, confidence interval; $C I R S$, Cumulative Illness Rating Scale; $M C C$, multiple chronic conditions 
Table 3 Odds ratio for in-hospital mortality, 30-day mortality, and 30-day readmission in patients with comorbidity vs multimorbidity, derived from a logistic model

\begin{tabular}{lllll}
\hline & & Odds ratio & $95 \%$ CI & $p$ value \\
\hline In-hospital mortality & $N(\%)$ & & & 0.533 \\
No MCC & $2(3.8)$ & 1 & & \\
Comorbidity & $22(7.7)$ & 2.12 & $0.48-9.32$ & 0.318 \\
Multimorbidity & $68(6.4)$ & 1.76 & $0.42-7.37$ & 0.441 \\
30-day mortality & & & & 0.088 \\
No MCC & $2(3.8)$ & 1 & & \\
Comorbidity & $43(15.0)$ & 4.51 & $1.06-19.23$ & 0.042 \\
Multimorbidity & $130(12.3)$ & 3.58 & $0.86-14.90$ & 0.079 \\
30-day readmission & & & & 0.227 \\
No MCC & $6(11.7)$ & 1 & & \\
Comorbidity & $44(22.7)$ & 2.20 & $0.88-5.50$ & 0.091 \\
Multimorbidity & $157(21.9)$ & 2.11 & $0.88-5.03$ & 0.093 \\
\hline
\end{tabular}

$C I$, confidence interval; $M C C$, multiple chronic conditions

mortality and 30-day readmission, while comorbidity was significantly associated with 30-day mortality (OR 4.51, $p=0.042$ ).

The multivariable analysis for the outcomes in-hospital mortality, 30-day mortality, and 30-day readmission outcomes are reported in Table 4. No differences were noticed for the comorbidity and multimorbidity categories and for gender. Regarding age, groups $75-84$ and $\geq 85$ were significantly associated with greater odds for in-hospital mortality (4.82 and 6.15, respectively) and 30-day mortality (3.42 and 6.20, respectively). An active oncological disease was present in only one (1.9\%) patient with no MCC, in 88 patients (30.8\%) with comorbidity, and in 81 patients $(7.7 \%)$ with multimorbidity (data not shown in the Table). When considering the specific ICD9 chapters, only chapter 2 (oncological diseases) maintained its significance after the Bonferroni correction (odds ratio 2.59; $p<0.001$ ) for 30-day mortality. Regarding the 30-day readmission outcome, no differences were noticed among the three categories no MCC, comorbidity, and multimorbidity, as well as no gender differences having emerged. Polypharmacy was significantly associated with increased odds for 30-day readmission (OR 1.34), as well as the ICD9 chapter 2 (OR 4.23), even after the Bonferroni correction $(p<0.001)$.

Given the potential negative impact of oncological diseases, we performed an alternative multivariable analysis excluding oncological patients (presenting an ICD9 chapter 2 disease). Even in this case, the comorbidity and multimorbidity groups were not associated, in a multivariable model, with any of the outcomes (in-hospital mortality, 30-day mortality and 30-day readmission; data not shown).

\section{Discussion}

MCC constitutes a major health problem, their prevalence is very high, both in the general population and in hospital settings [25-27], and this burden seems to be constantly growing [28]. Certainly, MCC heavily affect important outcomes, such as mortality [29] and healthcare utilization and costs [30]. Despite the above and taking into account the sharp increase of dedicated studies [31], there persists a confused conceptualization of the terms to describe the occurrence of MCC [7]. In this regard, the recently proposed distinction between comorbidity [8], defined in accordance with the seminal statement by Feinstein [32] as in relation to an index disease, and multimorbidity [9], in which no disease holds priority, represents an important achievement [10]. To assess whether this distinction has actual consistency in an internal medicine setting, we have used this conceptualization for the first time to evaluate how this is correlated with a series of demographic and clinical variables.

Considering all the 1394 inpatients, the prevalence of MCC (96.2\%) was higher than that reported in the general practice, $[33,34]$ but similar to what was found in previous hospital studies, which are, however, partly comparable to ours. In fact, even within the same setting, other differences affect generalizability of the data. Aubert and colleagues conducted a multicenter and multinational study, reporting a prevalence of MCC of $86 \%$ [35]. Most of the centers were academic, but also specialist units were included which might have lowered patients' median age (64 years). Moreover, the use of administrative data collected in a retrospective fashion may have affected the overall accuracy of that study [36]. A setting that has greater similarity to ours is that of Schneider and colleagues who found a prevalence of MCC of $93 \%$, although, also in this case administrative data were assessed [37]. In our study, the median age of patients with MCC was 78 years and patients aged 18-64 were under-represented compared to the primary care setting or to the general population $[33,34]$. Finally, in line with an Italian registry study, no gender predominance was found in patients with MCC [38].

As expected, and in keeping with all the previous literature [39-42], patients with MCC had, compared to those with only one acute or chronic disease, greater age, and worse outcomes, despite numerically unbalanced groups.

By dividing the patients into the three categories, namely no MCC, comorbidity, and multimorbidity, the latter turned out to be the most prevalent $(75.7 \%)$. This finding has no support in the literature, as no one has ever designed a similar study before. The median age of patients with multimorbidity was significantly higher 


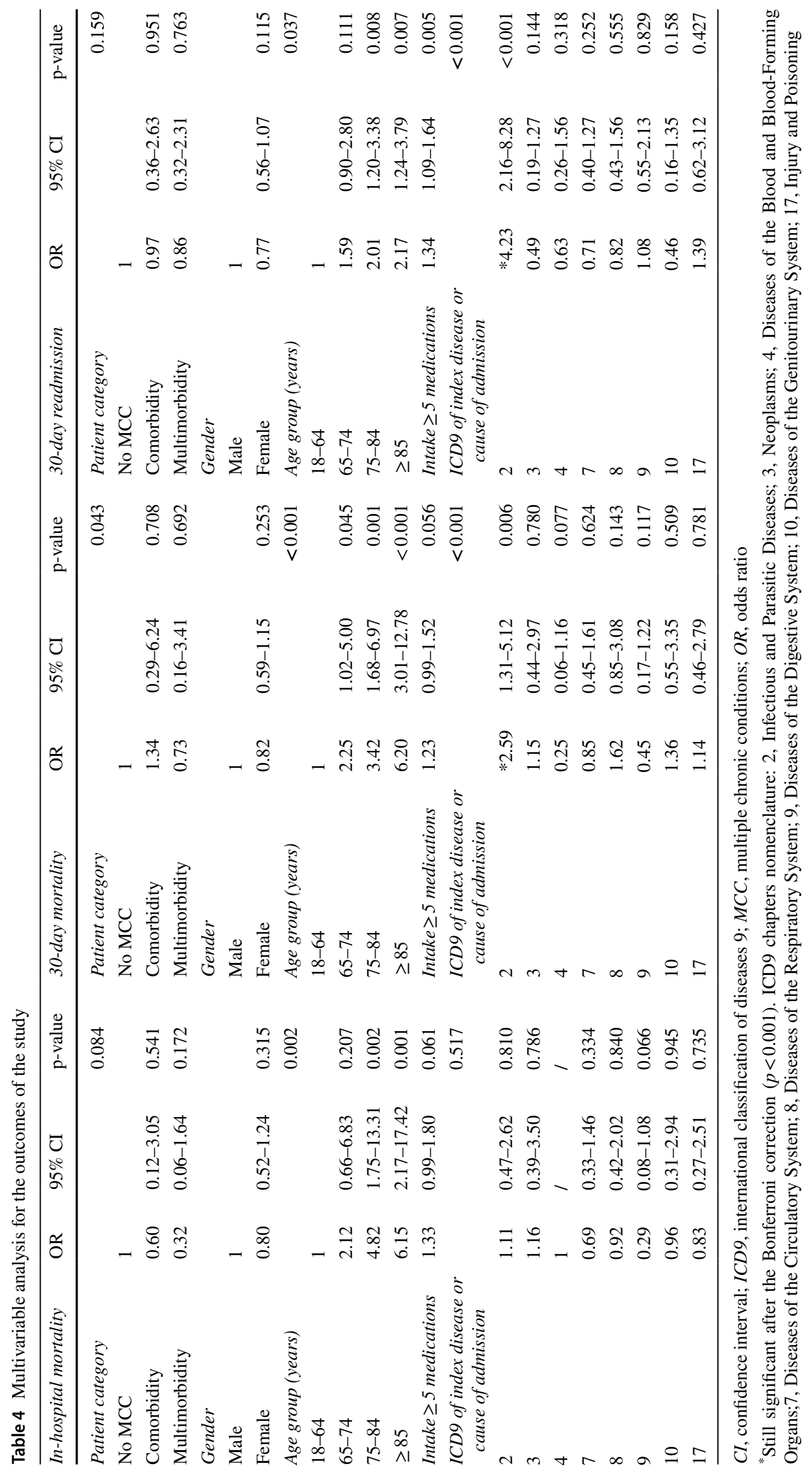


compared to those with no MCC and comorbidity. Moreover, a significantly increasing age trend was noticed, having multimorbidity the highest prevalence in patients aged 75 and over. This argues in favor of a stochastic accumulation of diseases, among which no disease holds priority, as a consequence of aging which carries epigenetic effects due to cumulative life events, prolonged impact of common risk factors and unhealthy lifestyle, frailty (which was actually more prevalent in this group), socioeconomic distress, and mental impairment [34, 43, 44]. Conversely, in comorbid patients (20.5\%) an opposite age trend was noticed. Of note, according to a recent systematic review, while most frail patients were also multimorbid (roughly $72 \%$ ), only a minority of multimorbid patients were frail (roughly 16\%) [45]. This figure is in line with our data, which reported a prevalence of frailty of roughly $19 \%$ in multimorbid patients. The prevalence of comorbidity instead was higher in the 18-64 age group and decreased with aging. Mechanisms other than aging, such as common genetic susceptibility [46], direct causation of a disease to another or the co-presence of a glaring complication especially when a chronological link is evident [43], could point at an index disease, and hence comorbidity [47]. Additionally, an etiological link between diseases may be more easily recognizable in younger patients, given the milder effect of aging.

A relevant result-being the primary endpoint of the study-is that multimorbid patients had greater CIRS comorbidity and severity indexes compared to comorbid patients. Notably, the median number of chronic diseases did not statistically differ between the two categories, confirming that this parameter did not interfere with patient categorization. After adjustment for age groups, this difference was significant only in patients aged $>75$, thus confirming an age effect not only in underlying patient categorization, but also in the magnitude of the CIRS scores.

Concerning polypharmacy, we have applied its most common definition, i.e., the intake of five or more medications [48]. Multimorbidity and polypharmacy are indeed strictly related, but the detrimental effects of drug-drug interactions are often overlooked [49]. Richardson and Doster, placing comorbidity and multimorbidity in the context of a three-dimensional framework of risk, responsiveness and vulnerability, predicted a different decision making about treatments in relation to these categories [12] and it has been said that concordant diseases may be managed synergistically [50]. Our results show that polypharmacy was more common in patients with multimorbidity $(78.7 \%)$ and it was significantly associated with a higher risk of 30-day readmission. Yet, roughly half (50.9\%) of the patients with comorbidity also had polypharmacy, and this may reflect the co-presence of concordant diseases which may lower the need for different classes of medications. The greater odds for 30-day readmission in polypharmacy (OR 1.34) could reflect the presence of more severe or unstable conditions requiring hospital admission.

Rather disappointingly, we did not find any meaningful differences in patients' major outcomes between the comorbidity and the multimorbidity frameworks, not even after the exclusion of neoplastic patients. However, also in a primary care practice, comorbidity scores did not predict important clinical outcomes, such as hospital admission and re-admissions, whereas different results were noticed when considering physician-defined complexity, that also took into account contextual and psychosocial contributors [51]. After all, it is well known that the available measures for categorizing MCC do not reflect the overall patients' complexity [14, 38, $43,52,53]$. Age once again confirmed to be the most important and independent determinant of in-hospital mortality, 30-day mortality, and 30-day readmission.

Indeed, our study has some limitations. Although we followed the MeSH criteria for differentiating comorbidity from multimorbidity, and although this differentiation was performed by a single expert physician, we are aware that the nuanced definitions may imply some subjectivity, which, however, reflects what happens in the real-world clinical practice. In addition, we did not take into account the allocation of diagnostic and therapeutical resources per each patient, which might be useful for identifying comorbidity and multimorbidity in clinical practice. The generalizability of our results is limited to an academic, internal medicine setting, and cannot be applied to the primary care setting, where the definition of an index disease might be more challenging. The brief follow-up time might have mitigated some differences in terms of major outcomes. On the other hand, our study also has many strengths, including the homogeneity of data, the real-world setting, and its monocentric nature. More importantly, all patients' data have been collected in a prospective fashion, through direct interview, without using administrative data which are hampered by many limitations [36]. The hospital setting guarantees, more than others, that all morbid conditions have been taken into account. Finally, this study was conducted just before the beginning of the SARS-CoV-2 pandemic, hence our results were not affected by this event.

To conclude, we have performed the first ad hoc study focusing on possible differences between patients with comorbidity and multimorbidity. Multimorbid patients are much more prevalent, older, and with a greater disease burden and polypharmacy. Most of the heterogeneity between multimorbidity and comorbidity was found to be a consequence of aging that, as expected, proved to be an independent determinant of mortality and re-hospitalization. In future studies, differentiating patients into these two frameworks may be useful in both research and clinical settings. 
Supplementary Information The online version contains supplementary material available at https://doi.org/10.1007/s11739-021-02899-2.

Acknowledgements Dr. Marco Vincenzo Lenti is grateful to the University of Pavia for supporting his research projects. We thank Intermediate srl (Rome, Italy) for having proofread the paper.

Author contributions All authors participated in the drafting of the manuscript or critical revision of the manuscript for important intellectual content and provided approval of the final submitted version. Individual contributions are as follows: GRC designed the study, GRC, MVL, and CK organized data collection and drafted the manuscript; all other authors conducted the study and enrolled patients; CK, performed the statistical analysis and interpreted data; ADS supervised patient recruitment; GRC made the final critical revision for important intellectual content. All authors approved the final version of the paper.

Funding This research is part of a project for the study of clinical complexity (SMAC study) funded by San Matteo Hospital FoundationItalian Ministry of Health (Progetto di Ricerca Corrente 2017-PI Prof. Gino Roberto Corazza). The funding source had no role in the design, execution, analyses, and interpretation of the data.

\section{Declarations}

Conflict of interest The authors have no conflicts of interest to declare.

Ethics approval and consent to participate This study was conducted according to the Declaration of Helsinki and the guidelines of Good Clinical Practice (World Medical Association, 2013). Written informed consent was obtained from patients, or the relatives/next of kin of critically ill patients, prior to enrolment. The study was approved by the local ethics committees of the participating hospitals (IRCCS San Matteo Hospital Foundation, protocol number 2017/0019414).

Human and animal rights All procedures performed in the study were in accordance with the ethical standards of the institutional and/or national research committee and with the 1964 Helsinki Declaration and its later amendments or comparable ethical standards.

\section{References}

1. Christensen K, Doblhammer G, Rau R, Vaupel JW (2009) Ageing populations: the challenges ahead. Lancet 374:1196-1208

2. The Lancet (2018) Making more of multimorbidity: an emerging priority. Lancet 391:1637

3. Fortin M, Soubhi H, Hudon C, Bayliss EA, van den Akker M (2007) Multimorbidity's many challenges. BMJ 334:1016-1017

4. Parekh AK, Barton MB (2010) The challenge of multiple comorbidity for the US health care system. JAMA 13:1303-1304

5. Xu X, Mishra GD, Jones M (2017) Evidence on multimorbidity from definition to intervention: An overview of systematic reviews. Ageing Res Rev 37:53-68

6. van den Akker M, Buntinx F, Knottnerus JA (1996) Comorbidity or multimorbidity. Eur J Gen Pract 2:65-70

7. Almirall J, Fortin M (2013) The coexistence of terms to describe the presence of multiple concurrent diseases. J Comorb 3:4-9

8. https://meshb-prev.nlm.nih.gov/record/ui?ui=D015897

9. https://www.ncbi.nlm.nih.gov/mesh/?term=multimorbidity

10. Tugwell P, Knottnerus JA (2019) Multimorbidity and comorbidity are now separate MESH headings. J Clin Epidemiol. https://doi. org/10.1016/j.jclinepi.2018.11.019 ([Epub ahead of print])
11. Nicholson K, Makovski TT, Griffith LE, Raina P, Stranges S, van den Akker M (2019) Multimorbidity and comorbidity revisited: refining the concepts for international health research. J Clin Epidemiol 105:142-146

12. Richardson WS, Doster LM (2014) Comorbidity and multimorbidity need to be placed in the context of a framework of risk, responsiveness, and vulnerability. J Clin Epidemiol 67:244-246

13. Dekker J, Buurman BM, van der Leeden M (2019) Exercise in people with comorbidity or multimorbidity. Health Psychol 38:822-830

14. Safford MM, Allison JJ, Kiefe CI (2007) Patient complexity: more than comorbidity. the vector model of complexity. J Gen Intern Med 22:382-390

15. Corazza GR, Klersy C, Formagnana P, Lenti MV, Padula D, Consensus Panel (2017) A consensus for the development of a vector model to assess clinical complexity. Intern Emerg Med 12:1313-1318

16. Lenti MV, Klersy C, Brera AS et al (2019) Reproducibility in the assessment of the components of a clinical complexity index. $\mathrm{J}$ Gen Intern Med 34:2316-2318

17. Lenti MV, Klersy C, Brera AS et al (2020) Clinical complexity and hospital admissions in the December holiday period. PLoS ONE 15:e0234112

18. Rolfson DB, Majumdar SR, Tsuyuki RT, Tahir A, Rockwood K (2006) Validity and reliability of the Edmonton Frail Scale. Age Ageing 35:526-529

19. Linn BS, Linn MW, Gurel L (1968) Cumulative illness rating scale. J Am Geriatr Soc 16:622-626

20. Johnston MC, Crilly M, Black C, Prescott GJ, Mercer SW (2019) Defining and measuring multimorbidity: a systematic review of systematic reviews. Eur J Public Health 29:182-189

21. Stirland LE, González-Saavedra L, Mullin DS, Ritchie CW, Muniz-Terrera G, Russ TC (2020) Measuring multimorbidity beyond counting diseases: systematic review of community and population studies and guide to index choice. BMJ 368:m160

22. Salvi F, Miller MD, Grilli A et al (2008) A manual of guidelines to score the modified cumulative illness rating scale and its validation in acute hospitalized elderly patients. J Am Geriatr Soc 56:1926-1931

23. https://www.ncbi.nlm.nih.gov/mesh/81000150

24. https://www.ncbi.nlm.nih.gov/mesh?Db=mesh\&Cmd=DetailsSea rch\&Term $=\% 22$ Chronic+Disease $\% 22 \% 5 \mathrm{BMeSH}+$ Terms $\% 5 \mathrm{D}$

25. van den Akker M, Buntinx F, Metsemakers JF, Roos S, Knottnerus JA (1998) Multimorbidity in general practice: prevalence, incidence, and determinants of co-occurring chronic and recurrent diseases. J Clin Epidemiol 51:367-375

26. Mannucci PM, Nobili A, Investigators REPOSI (2014) Multimorbidity and polypharmacy in the elderly: lessons from REPOSI. Intern Emerg Med 9:723-734

27. Fortin M, Stewart M, Poitras ME, Almirall J, Maddocks H (2012) A systematic review of prevalence studies on multimorbidity: toward a more uniform methodology. Ann Fam Med 10:142-151

28. Pefoyo AJ, Bronskill SE, Gruneir A et al (2015) The increasing burden and complexity of multimorbidity. BMC Public Health $15: 415$

29. Lee TA, Shields AE, Vogeli C et al (2007) Mortality rate in veterans with multiple chronic conditions. J Gen Intern Med 22:403-407

30. Hajat C, Stein E (2018) The global burden of multiple chronic conditions: a narrative review. Prev Med Rep 12:284-293

31. Salisbury C (2013) Multimorbidity: time for action rather than words. Br J Gen Pract 63:64-65

32. Feinstein AR (1970) The pre-therapeutic classification of comorbidity in chronic disease. J Chronic Dis 23:455-468

33. van den Akker M, Buntinx F, Metsemakers JFM, Roos S, Knottnerus JA (1998) Multimorbidity in general practice: 
prevalence, incidence, and determinants of co-occurring chronic and recurrent diseases. J Clin Epidemiol 51:365-375

34. Barnett K, Mercer SW, Norbury M, Watt G, Wyke S, Guthrie B (2012) Epidemiology of multimorbidity and implications for health care, research, and medical education: a cross-sectional study. Lancet 380:37-43

35. Aubert CE, Schnipper JL, Fankhauser N et al (2020) Patterns of multimorbidity in medical inpatients: a multinational retrospective cohort study. Intern Emerg Med 15:1207-1217

36. Lenti MV, Corazza GR (2020) Administrative data for exploring multimorbidity in hospitalised patients. Intern Emerg Med 15:1161-1163

37. Schneider F, Kaplan V, Rodak R, Battegay E, Holzer B (2012) Prevalence of multimorbidity in medical inpatients. Swiss Med Wkly 142:w13533

38. Corrao S, Natoli G, Nobili A, RePoSI Investigators et al (2020) Comorbidity does not mean clinical complexity: evidence from the RePoSI register. Intern Emerg Med 15:621-628

39. Lenzi J, Avaldi VM, Rucci P, Pieri G, Fantini MP (2016) Burden of multimorbidity in relation to age, gender and immigrant status: a cross-sectional study based on administrative data. BMJ Open 6:e012812

40. Sakib MN, Shooshtari S, St John P, Menec V (2019) The prevalence of multimorbidity and associations with lifestyle factors among middle-aged Canadians: an analysis of Canadian longitudinal study on aging data. BMC Public Health 19:243

41. Schäfer I, Kaduszkiewicz H, Nguyen TS, van den Bussche H, Scherer M, Schön G (2018) Multimorbidity patterns and 5-year overall mortality: results from a claims data-based observational study. J Comorb 8:2235042X18816588

42. Willadsen TG, Siersma V, Nicolaisdóttir DR et al (2018) Multimorbidity and mortality: a 15-year longitudinal registry-based nationwide Danish population study. J Comorb 8:2235042X18804063

43. Valderas JM, Starfield B, Sibbald B, Salisbury C, Roland M (2009) Defining comorbidity: implications for understanding health and health services. Ann Fam Med 7:357-363

44. Violan C, Foguet-Boreu Q, Flores-Mateo G et al (2014) Prevalence, determinants and patterns of multimorbidity in primary care: a systematic review of observational studies. PLoS ONE 9:e102149

45. Vetrano DL, Palmer K, Marengoni A et al (2019) Frailty and multimorbidity: a systematic review and meta-analysis. J Gerontol A Biol Sci Med Sci 74:659-666

46. Menche J, Sharma A, Kitsak M et al (2015) Disease networks. Uncovering disease-disease relationships through the incomplete interactome. Science 347:1257601

47. Gijsen R, Hoeymans N, Schellevis FG, Ruwaard D, Satariano WA, van den Bos GA (2001) Causes and consequences of comorbidity: a review. J Clin Epidemiol 54:661-674

48. Masnoon N, Shakib S, Kalisch-Ellett L, Caughey GE (2017) What is polypharmacy? A systematic review of definitions. BMC Geriatr 17:230

49. Boyd CM, Darer J, Boult C, Fried LP, Boult L, Wu AW (2005) Clinical practice guidelines and quality of care for older patients with multiple comorbid diseases: implications for pay for performance. JAMA 294:716-724

50. Boyd CM, Fortin M (2010) Future of multimorbidity research: How should understanding of multimorbidity inform health system design? Public Health Rev 32:451-474

51. Hong CS, Atlas SJ, Ashburner JM et al (2015) Evaluating a model to predict primary care physician-defined complexity in a large academic primary care practice-based research network. J Gen Intern Med 30:1741-1747

52. Grant RW, Ashburner JM, Hong CS, Chang Y, Barry MJ, Atlas SJ (2011) Defining patient complexity from the primary care physician's perspective: a cohort study. Ann Intern Med 155:797-804

53. Corazza GR, Formagnana P, Lenti MV (2019) Bringing complexity into clinical practice: an internistic approach. Eur J Intern Med 61:9-14

Publisher's Note Springer Nature remains neutral with regard to jurisdictional claims in published maps and institutional affiliations. 